\title{
Well-Composed Cell Complexes
}

\author{
Rocio Gonzalez-Diaz, Maria-Jose Jimenez, and Belen Medrano
}

Applied Math Department, University of Seville, Av. Reina Mercedes, s/n, Seville, Spain

\{rogodi, majiro, belenmg\}@us.es

\begin{abstract}
Well-composed 3D digital images, which are 3D binary digital images whose boundary surface is made up by $2 \mathrm{D}$ manifolds, enjoy important topological and geometric properties that turn out to be advantageous for some applications. In this paper, we present a method to transform the cubical complex associated to a 3D binary digital image (which is not generally a well-composed image) into a cell complex that is homotopy equivalent to the first one and whose boundary surface is composed by $2 \mathrm{D}$ manifolds. This way, the new representation of the digital image can benefit from the application of algorithms that are developed over surfaces embedded in $\mathbb{R}^{3}$.
\end{abstract}

Keywords: Well-composed digital images, cubical complex, cell complex, homotopy equivalence.

\section{Introduction}

We are mainly interested in studying topological features of $3 \mathrm{D}$ digital images. More concretely, our ultimate purpose is that of extracting cohomological information of a 3D model that could be used in characterization or recognition tasks (see [5647] as related works). For this aim, it would be useful to compute first geometrically relevant representative cycles of homology generators of dimension 1 in the surface of the model, since this could simplify cohomological computations. Many applications such as topology repair, surface parameterization and feature recognition benefit from computing loops on surfaces that wrap around their 'handles' and 'tunnels' (defined by Dey et al. in [2]). In the paper [3], there is an algorithm to compute topologically correct loops that are also geometrically relevant in that sense. A refinement of the algorithm is given in [1]. However, all the computations are carried out over a connected closed surface in $\mathbb{R}^{3}$. Since we are interested in applying these results to 3D binary digital images, we focus in the so-called well-composed images. A 3D binary digital image is said to be well-composed if and only if the square faces shared by foreground and background voxels of the image form a 2D manifold. Well-composed images enjoy important topological and geometric properties: there is only one type of connected component in any well-composed image, and hence, several algorithms used in computer vision, computer graphics and image processing are simpler; thinning algorithms can be simplified and naturally made parallel if the input 
image is well-composed [1013]; some algorithms for computing surface curvature or extracting adaptive triangulated surfaces [8], assume that the input image is well-composed. Since 2D and 3D images are often not well-composed images, there are several methods (repairing algorithms) for turning 3D binary digital images that are not well-composed into well-composed ones (see [12 15]), but these methods do not guarantee the topological equivalence between the original object and its corresponding well-composed image. In fact, the purpose can even be to simplify as much as possible the topology in the sense of removing little topological artifacts from the image. However we are concerned with the fact of preserving the topology of the input image having in mind cases in which subtle details can be important.

\section{3D Digital Images and Cubical Complexes}

Consider $\mathbb{Z}^{3}$ as the set of points with integer coordinates in $3 \mathrm{D}$ space $\mathbb{R}^{3}$. A $3 \mathrm{D}$ binary digital image $I=\left(\mathbb{Z}^{3}, 26,6, B\right)$ (or $I=\left(\mathbb{Z}^{3}, B\right)$ for short), where $B \subset \mathbb{Z}^{3}$ is the foreground and $B^{c}=\mathbb{Z}^{3} \backslash B$ the background, is represented by the set of unit cubes (voxels) centered at the points of $B$ together with all their faces. This identification of voxels with $3 \mathrm{D}$ cubes in $\mathbb{R}^{3}$ leads, in a natural way, to the combinatorial structure of cubical complexes, whose geometric building blocks (cells) are points, edges, squares and cubes (see [9]). More concretely, given a voxel centered at a point of $\mathbb{Z}^{3}$ of coordinates $(i, j, k)$, the cells associated to this voxel considered as a 3D cube are denoted as follows (see Fig 1):

- The eight vertices (0-cells): $\left(i \pm \frac{1}{2}, j \pm \frac{1}{2}, k \pm \frac{1}{2}\right)$

- The twelve edges (1-cells): $\left(i, j \pm \frac{1}{2}, k \pm \frac{1}{2}\right),\left(i \pm \frac{1}{2}, j, k \pm \frac{1}{2}\right),\left(i \pm \frac{1}{2}, j \pm \frac{1}{2}, k\right)$.

- The six square faces (2-cells): $\left(i, j, k \pm \frac{1}{2}\right),\left(i, j \pm \frac{1}{2}, k\right),\left(i \pm \frac{1}{2}, j, k\right)$.

- The cube (3-cell): $(i, j, k)$.

By considering the $(26,6)$-relationship we can guarantee that the topology of the cubical complex associated to the image reflects the topology of the object.

A cubical complex is, in fact, a special case of cell complex, which is a more general topological structure by which a space is decomposed into basic elements (cells) of different dimensions that are glued together by their boundaries.

Given a cell complex $K$, a proper face of $\sigma \in K$ is a face of $\sigma$ whose dimension is strictly less than the one of $\sigma$. A facet of $\sigma$ is a proper face of $\sigma$ of maximal dimension. A maximal cell of $K$ is a cell of $K$ which is not a proper face of any other cell of $K$. The dimension of $K$ is the maximal dimension of the maximal cell of $K$. A facet that is incident only to one cell is called a boundary facet. The union of all the boundary facets is the boundary of the cell complex which is denoted by $\partial K$.

The cubical complex $Q(I)$ representing a 3D binary digital image $I$ satisfies that the maximal cells are cubes and the elements of the boundary of $Q(I)$, $\partial Q(I)$, are all the square faces of $Q(I)$ which are shared by a voxel of $B$ and a voxel of $B^{c}$ together with all their faces. 


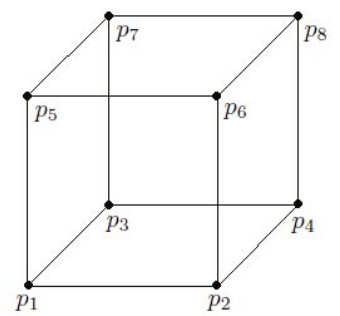

$$
\begin{array}{ll}
p_{1}=\left(i+\frac{1}{2}, j-\frac{1}{2}, k-\frac{1}{2}\right) & p_{5}=\left(i+\frac{1}{2}, j-\frac{1}{2}, k+\frac{1}{2}\right) \\
p_{2}=\left(i+\frac{1}{2}, j+\frac{1}{2}, k-\frac{1}{2}\right) & p_{6}=\left(i+\frac{1}{2}, j+\frac{1}{2}, k+\frac{1}{2}\right) \\
p_{3}=\left(i-\frac{1}{2}, j-\frac{1}{2}, k-\frac{1}{2}\right) & p_{7}=\left(i-\frac{1}{2}, j-\frac{1}{2}, k+\frac{1}{2}\right) \\
p_{4}=\left(i-\frac{1}{2}, j+\frac{1}{2}, k-\frac{1}{2}\right) & p_{8}=\left(i-\frac{1}{2}, j+\frac{1}{2}, k+\frac{1}{2}\right)
\end{array}
$$

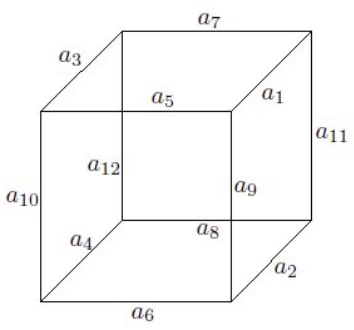

$$
\begin{array}{lll}
a_{1}=\left(i, j+\frac{1}{2}, k+\frac{1}{2}\right) & a_{5}=\left(i+\frac{1}{2}, j, k+\frac{1}{2}\right) & a_{9}=\left(i+\frac{1}{2}, j+\frac{1}{2}, k\right) \\
a_{2}=\left(i, j+\frac{1}{2}, k-\frac{1}{2}\right) & a_{6}=\left(i+\frac{1}{2}, j, k-\frac{1}{2}\right) & a_{10}=\left(i+\frac{1}{2}, j-\frac{1}{2}, k\right) \\
a_{3}=\left(i, j-\frac{1}{2}, k+\frac{1}{2}\right) & a_{7}=\left(i-\frac{1}{2}, j, k+\frac{1}{2}\right) & a_{11}=\left(i-\frac{1}{2}, j+\frac{1}{2}, k\right) \\
a_{4}=\left(i, j-\frac{1}{2}, k-\frac{1}{2}\right) & a_{8}=\left(i-\frac{1}{2}, j, k-\frac{1}{2}\right) & a_{12}=\left(i-\frac{1}{2}, j-\frac{1}{2}, k\right)
\end{array}
$$

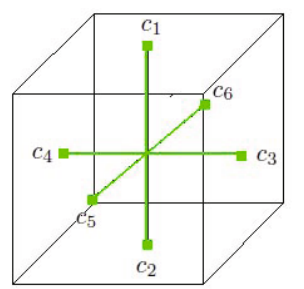

$$
\begin{array}{ll}
c_{1}=\left(i, j, k+\frac{1}{2}\right) & c_{4}=\left(i, j-\frac{1}{2}, k\right) \\
c_{2}=\left(i, j, k-\frac{1}{2}\right) & c_{5}=\left(i+\frac{1}{2}, j, k\right) \\
c_{3}=\left(i, j+\frac{1}{2}, k\right) & c_{6}=\left(i-\frac{1}{2}, j, k\right)
\end{array}
$$

Fig. 1. Notation of the vertices $\left\{p_{i}\right\}$, edges $\left\{a_{i}\right\}$ and square faces $\left\{c_{i}\right\}$ associated to the voxel $(i, j, k)$

Given a cell complex $K$, define $K_{q}$ as the set of $q$-cells of $K$. Define the morphism $k_{q}: K_{q} \times K_{q-1} \rightarrow \mathbb{Z}_{2}$ given by $k_{q}\left(c, c^{\prime}\right):=1$ if $c^{\prime}$ is a facet of $c$ and $k_{q}\left(c, c^{\prime}\right):=0$ in other case (we do not take into account orientation). Then, we can codify a cell complex as a pair $(K, k)$ where $K=\bigcup_{q} K_{q}$ is the set of cells of $K$ and $k=\oplus_{q} k_{q}$ defines the relation between each cell and its facets. The morphism $k$ is called the incidence number (see [14]).

For example, since any cubical complex $Q$ is a particular case of cell complex, it can be codified as a pair $\left(Q, k^{Q}\right)$ : Let $Q_{0}$ be the set of vertices, $Q_{1}$ the set of edges, $Q_{2}$ the set of square faces and $Q_{3}$ the set of cubes of $Q$. Define the morphism $k_{q}^{Q}: Q_{q} \times Q_{q-1} \rightarrow \mathbb{Z}_{2}$ given by $k_{q}^{Q}\left(c, c^{\prime}\right):=1$ if $c^{\prime}$ is a facet of $c$ and $k_{q}^{Q}\left(c, c^{\prime}\right):=0$ in other case. Taking into account the notation given for the cells of $Q$ as points in $\mathbb{R}^{3}$, define $k_{q}^{Q}\left(c, c^{\prime}\right):=1$ if the Euclidean distance between $c$ and $c^{\prime}$ is $\frac{1}{2}$ and $k_{q}^{Q}\left(c, c^{\prime}\right):=0$ in other case.

\section{Well-Composed Cell Complexes}

A $3 D$ binary digital image $I=\left(\mathbb{Z}^{3}, B\right)$ is well-composed [10] if the boundary of the cubical complex associated, $\partial Q(I)$, is a $2 D$ manifold, i.e. if each point in 
$\partial Q(I)$ has a neighborhood homeomorphic to $\mathbb{R}^{2}$. This definition implies a simple correspondence between a $3 D$ binary digital image and the boundary surface of the associated cubical complex. Thus, one can use well-known properties of continuous boundary surfaces to determine and analyze properties of these digital images.

Since the boundary of the cubical complex associated to $I=\left(\mathbb{Z}^{3}, B\right)$ coincides with the one of $I^{c}=\left(\mathbb{Z}^{3}, B^{c}\right)$, a $3 D$ binary digital image $I$ is well-composed iff $I^{c}$ is also well-composed.

3D binary digital images are often not well-composed images. Nevertheless, there are several methods for turning 3D binary digital images that are not well-composed into well-composed ones (see [1215]). The problem is that, in general, these techniques do not guarantee the topological equivalence between the original object and its corresponding well-composed image, since they can modify the original image by moving some voxels from $B^{c}$ to $B$.

In this section, we are interested in obtaining a homotopy-equivalent cell complex to the cubical complex associated to a 3D binary digital image whose geometric realization could enjoy the advantages of well-composed images. Specifically, we present a method to transform the cubical complex $Q$ associated to a 3D binary digital image which is not generally a well-composed image into a cell complex $(K, k)$. This cell complex $(K, k)$ satisfies that it is homotopy equivalent to $Q$ and its boundary surface, $\partial K$, is composed by $2 \mathrm{D}$ manifolds.

The following proposition shows the characterization of well-composed images in terms of simple local conditions on cubes in the cubical complex associated, as one can observe in Fig. 2 ,

Proposition 1. [11] A $3 D$ binary digital image $I=\left(\mathbb{Z}^{3}, B\right)$ is well-composed iff the configurations of cubes C1, C2 and C3 (modulo reflections and rotations) do not occur in $Q(I)$ :

C1 Four cubes share an edge $(a, b, c)$ and exactly two of them which do not share a face are contained in $Q(I)$ and the other two are not contained in $Q(I)$. That is, if $W$ denotes the set of the four cubes sharing the edge $(a, b, c)$, there are exactly two cubes of $W$, denoted by $w_{1}$ and $w_{2}$, that are cubes of $Q(I)$ and whose squared Euclidean distance is 2 .

C2 Eight cubes share a vertex $(a, b, c)$ and exactly two of them which are corneradjacent are contained in $Q(I)$ while the other six are not. That is, if $S$ denotes the set of the eight cubes sharing the vertex $(a, b, c)$, there are exactly two cubes of $S$, denoted by $s_{1}$ and $s_{2}$, that are cubes of $Q(I)$ and whose squared Euclidean distance is 3 .

C3 Eight cubes share a corner point and exactly two of them which are corneradjacent are contained in $Q\left(I^{c}\right)$ while the other six are not. That is, if $T$ denotes the set of the eight cubes sharing the vertex $(a, b, c)$. Then, there are exactly two cubes of $T$, denoted by $t_{1}$ and $t_{2}$, that are not cubes of $Q(I)$ and whose squared Euclidean distance is 3 .

Given a cubical complex $Q$ associated to a 3D binary digital image, we say that an edge $(a, b, c)$ of $Q$ is a critical edge if $(a, b, c)$ is an edge shared by four 

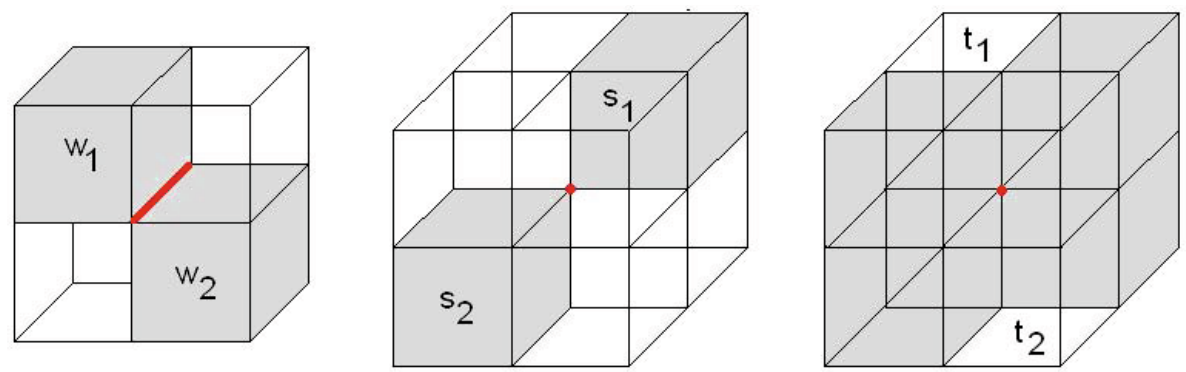

Fig. 2. Configurations C1, C2 and C3 (modulo reflections and rotations). These configurations cannot occur in $Q(I)$.

cubes that constitute the C1-configuration. We say that a vertex $(a, b, c)$ of $Q$ is a critical vertex if it is either a vertex shared by eight cubes that constitute the $\mathrm{C} 2$-configuration or a vertex shared by eight cubes that constitute the C3-configuration or a vertex shared by eight cubes such that one or several critical edges are incident to it. Summing up, one can observe in Fig. 3 all the critical configurations that are possible by the combination of the mentioned critical elements within a set of eight cubes sharing a vertex. Notice that configurations $C(2,0), C(2,1), C(3,0)$ and $C(3,1)$ are complementary to configurations $C(6,2)$, $C(6,1), C(5,1)$ and $C(5,2)$, respectively; as well as configurations $C(4,1), C(4,2)$ and $C(4,3)$ are self-complementary.

Now, given a cubical complex $Q$ associated to a 3D binary digital image, we present a method to generate a new cell complex by some basic operations on the input cubical complex to repair all the critical edges and critical vertices that appear in $Q$. The method consists of three steps: (1) all the critical edges and critical vertices that appear in $Q$ are labeled and put into either the set $E$ of critical edges or the set $V$ of critical vertices; (2) apply Algorithm 1 to the cubical complex $Q$ to repair all the edges of $E$ and (3) apply Algorithm 2 to the cell complex output by the previous algorithm to repair all the vertices of $V$.

Algorithm 1. Repair the critical edges in E.

InPUT: The cubical complex $\left(Q, k^{Q}\right)$ associated to a $3 D$ binary digital image.

Initialize $K_{i}^{\prime}:=\left\{(a, b, c, 0)\right.$ : such that $\left.(a, b, c) \in Q_{i}\right\}$ and $k_{i}^{\prime}\left((a, b, c, 0),\left(a^{\prime}, b^{\prime}, c^{\prime}, 0\right)\right)$ $:=k_{i}^{Q}\left((a, b, c),\left(a^{\prime}, b^{\prime}, c^{\prime}\right)\right)$, for any $(a, b, c),\left(a^{\prime}, b^{\prime}, c^{\prime}\right) \in Q_{i}$ and for $0 \leq i \leq 3$.

- For each critical edge $(a, b, c) \in E$, do:

- Duplicate the edge: $K_{1}^{\prime}:=K_{1}^{\prime} \backslash\{(a, b, c, 0)\} \cup\{(a, b, c, 1),(a, b, c, 2)\}$.

- Add a new 2-cell: $K_{2}^{\prime}:=K_{2}^{\prime} \cup\{(a, b, c, 1,2)\}$.

- Denote by $w_{11}$ and $w_{12}$ the two square faces of $w_{1}$ sharing the edge $(a, b, c)$. Denote by $w_{21}$ the one of the square faces of $w_{2}$ sharing the edge $(a, b, c)$ such that the squared Euclidean distance between $w_{11}$ and $w_{21}$ is $\frac{1}{2}$. Denote by $w_{22}$ the other square face of $w_{2}$ sharing the edge $(a, b, c)$ (see Fig. 使). 

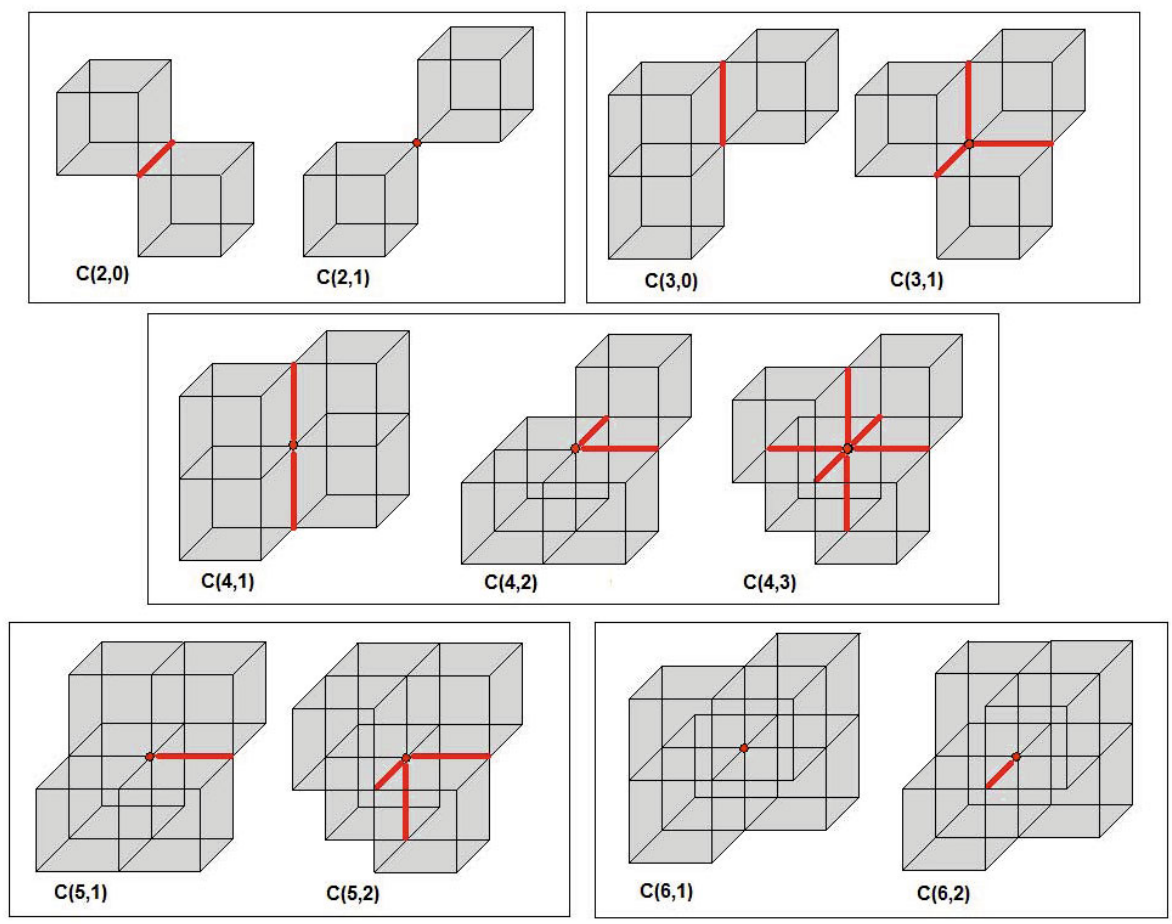

Fig. 3. All the critical configurations within a set of eight cubes sharing one vertex (modulo reflections and rotations)

- Define $k_{1}^{\prime}((a, b, c, j), v):=k_{1}^{\prime}((a, b, c, 0), v)$ for $j=1,2$ and for any vertex $v \in K_{0}^{\prime}$.

- Define $k_{2}^{\prime}(f,(a, b, c, j)):=1$ if $f=w_{i j}$, for $i=1,2$, or $f=(a, b, c, 1,2)$, for $j=1,2$.

- Define $k_{2}^{\prime}((a, b, c, 1,2), e):=0$ if $e \neq(a, b, c, j)$ for $j=1,2$, that is, the only facets of the new 2-cell $(a, b, c, 1,2)$ are the edges $(a, b, c, 1)$ and $(a, b, c, 2)$.

- Define $k_{3}^{\prime}(w,(a, b, c, 1,2)):=1$ if $w=w_{i}$ for $i=1,2$ and 0 in other case.

Output: The cell complex $\left(K^{\prime}, k^{\prime}\right)$.

Algorithm 2. Repair the critical vertices in $V$.

INPUT: The cell complex $\left(K^{\prime}, k^{\prime}\right)$ obtained after applying Algorithm 1.

Initialize $K_{i}:=K_{i}^{\prime}$ and $k_{i}:=k_{i}^{\prime}$ for $0 \leq i \leq 3$.

- For each critical vertex $(a, b, c) \in V$ with the configuration $C(2,1)$, do:

- Duplicate the vertex: $K_{0}:=K_{0} \backslash\{(a, b, c, 0)\} \cup\{(a, b, c, 1),(a, b, c, 2)\}$.

- Add two new edges: $\left.K_{1}:=K_{1} \cup\{(a, b, c, 1,2),(a, b, c, 2,1))\right\}$.

- Add a new 2-cell: $K_{2}:=K_{2} \cup\{(a, b, c, 1,2,1)\}$. 

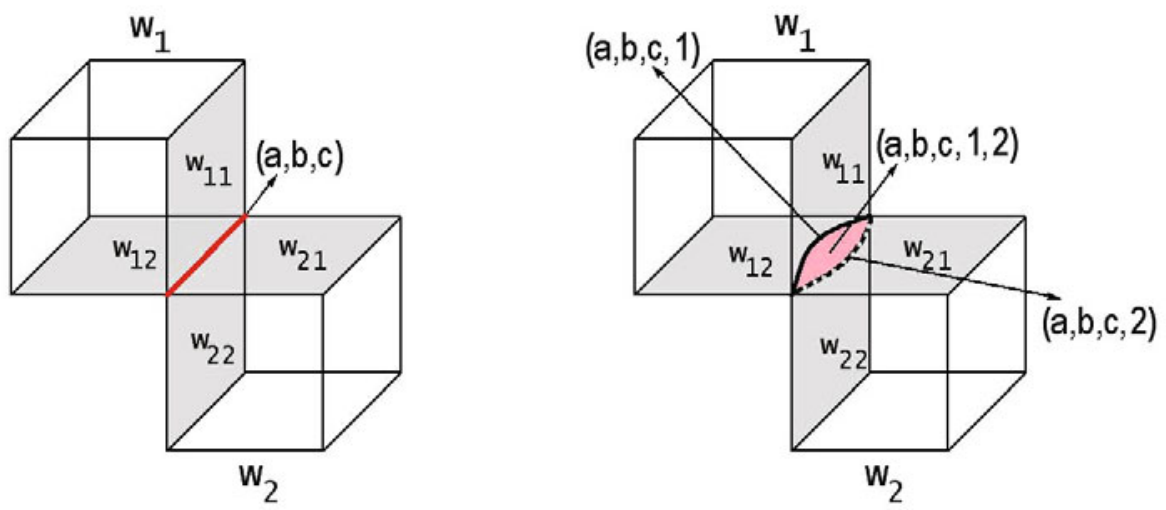

Fig. 4. Notations for the cells around a critical edge $(a, b, c)$

- Take two square faces of $s_{1}$ sharing the vertex $(a, b, c)$ and denote them by $s_{11}$ and $s_{12}$. Take the square face of $s_{2}$ sharing the vertex $(a, b, c)$ whose squared Euclidean distance to $s_{11}$ is 2 ; denote it by $s_{22}$. Denote by $s_{21}$ the other square face of $s_{2}$ sharing the vertex $(a, b, c)$ whose squared Euclidean distance to $s_{12}$ is 2 (see Fig. 5).

- Define $k_{1}(e,(a, b, c, 1)):=1$ if either $e=(a, b, c, 1,2)$ or $e=(a, b, c, 2,1)$, or $e$ is a face edge of $s_{1}$ shared by $s_{11}$ and $s_{12}$ or $e$ is any of the other two edges of $s_{2}$ such that $k_{1}^{\prime}(e,(a, b, c, 0))=1$ and that are not shared by $s_{21}$ and $s_{22}$ at the same time, and 0 otherwise.

- Define $k_{1}(e,(a, b, c, 2)):=1$ if either $e=(a, b, c, 1,2)$ or $e=(a, b, c, 2,1)$, or $e$ is a face edge of $s_{2}$ shared by $s_{21}$ and $s_{22}$ or $e$ is any of the other two edges of $s_{1}$ such that $k_{1}^{\prime}(e,(a, b, c, 0))=1$ and that are not shared by $s_{11}$ and $s_{12}$ at the same time, and 0 otherwise.

- Define $k_{2}(f,(a, b, c, 1,2)):=1$ if $f=s_{12}$ or $f=s_{22}$ or $f=(a, b, c, 1,2,1)$, and 0 otherwise.

- Define $k_{2}(f,(a, b, c, 2,1)):=1$ if $f=s_{21}$ or $f=s_{11}$ or $f=(a, b, c, 1,2,1)$, and 0 otherwise.

- Define $k_{3}(w,(a, b, c, 1,2,1)):=1$ if $w=s_{i}$ for $i=1,2$ and 0 otherwise.

- For each critical vertex $(a, b, c) \in V$ with any configuration of Fig. 3 apart from $C(2,1)$, do:

- Label the background connected components of the configuration of eight 3-cells around the critical vertex with labels 1, 2, 3 and 4, respectively (notice that the number of connected components may vary from 2 to 4 ). Let $L=\{1,2, \ldots, l\}$ be the set of labels assigned.

- Label all the edges incident to $(a, b, c)$ that belong to the boundary of $K$, with the corresponding label of the background component that shares such an edge.

- Substitute the critical vertex by a set of vertices, one for each label corresponding to a background connected component: $K_{0}:=K_{0} \backslash\{(a, b, c, 0)\} \cup$ $\{(a, b, c, 1), \ldots,(a, b, c, l)\}$. 


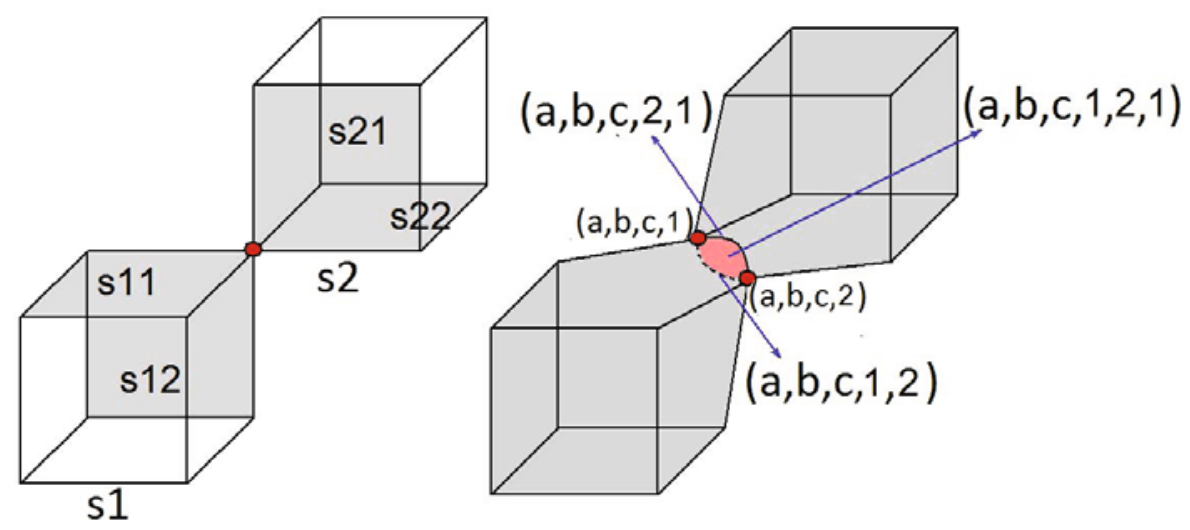

Fig. 5. Notations for the cells around a critical vertex $(a, b, c)$ with the configuration $C(2,1)$

- Add new edges: $K_{1}:=K_{1} \cup\{(a, b, c, i, j), i, j \in L, i<j\}$.

- If $l \geq 3$, add new 2-cells $K_{2}:=K_{2} \cup\{(a, b, c, i, j, k), i, j, k \in L, i<j<$ $k\}$.

- If $l=4$, add a new 3-cell $K_{3}:=K_{3} \cup\{(a, b, c, 1,2,3,4)\}$.

- Define $k_{1}(e,(a, b, c, i)):=1$ if either $k_{1}^{\prime}(e,(a, b, c, 0))=1$ and $e$ is an edge with label $i$ or $e=(a, b, c, i, j)$, for any $j$ or $e=(a, b, c, j, i)$, for any $j$, and 0 otherwise.

- Define $k_{2}(f,(a, b, c, i, j)):=1$ if either $f$ is a 2-cell with two face edges labeled as $i$ and $j$ or $f=(a, b, c, i, j, k)$ or $f=(a, b, c, i, k, j)$ or $f=$ $(a, b, c, k, i, j)$, and 0 otherwise.

- Define $k_{3}(w,(a, b, c, i, j, k)):=1$ if either $w$ is a 3-cell with three face edges labeled as $i, j$ and $k$, or $w=(a, b, c, 1,2,3,4)$, and 0 otherwise.

Output: The cell complex $(K, k)$.

Proposition 2. The cell complex $(K, k)$ and the cubical complex $Q$ are homotopy equivalent. Moreover, the boundary surface $\partial K$, of the cell complex $(K, k)$, is composed by $2 D$ manifolds, that is, each point of $\partial K$ has a neighborhood homeomorphic to $\mathbb{R}^{2}$.

Example 1. Consider the $3 D$ binary digital image $I=\left(\mathbb{Z}^{3}, B\right)$ with $B=\{(1,1,0)$, $(0,0,1),(0,2,1),(0,1,2)\}$. Let $Q$ the cubical complex associated to the image. This cubical complex has 4 voxeles (3-cells), 24 square faces (2-cells), 45 edges (1-cells) and 26 vertices (0-cells). The conflictive cells of $K$ are:

- The edges $a_{1}=\left(0, \frac{1}{2}, \frac{3}{2}\right)$ and $a_{2}=\left(0, \frac{3}{2}, \frac{3}{2}\right)$;

- The vertices $v_{1}=\left(\frac{1}{2}, \frac{1}{2}, \frac{1}{2}\right)$ and $v_{2}=\left(\frac{1}{2}, \frac{3}{2}, \frac{1}{2}\right)$.

Applying the previous method to this cubical complex, the obtained wellcomposed cell complex $K$ have four 3-cells, twenty eight 2-cells (21 square faces, 2 pentagons and 1 hexagon), 51 edges and 28 vertices (see Fig. (7). 

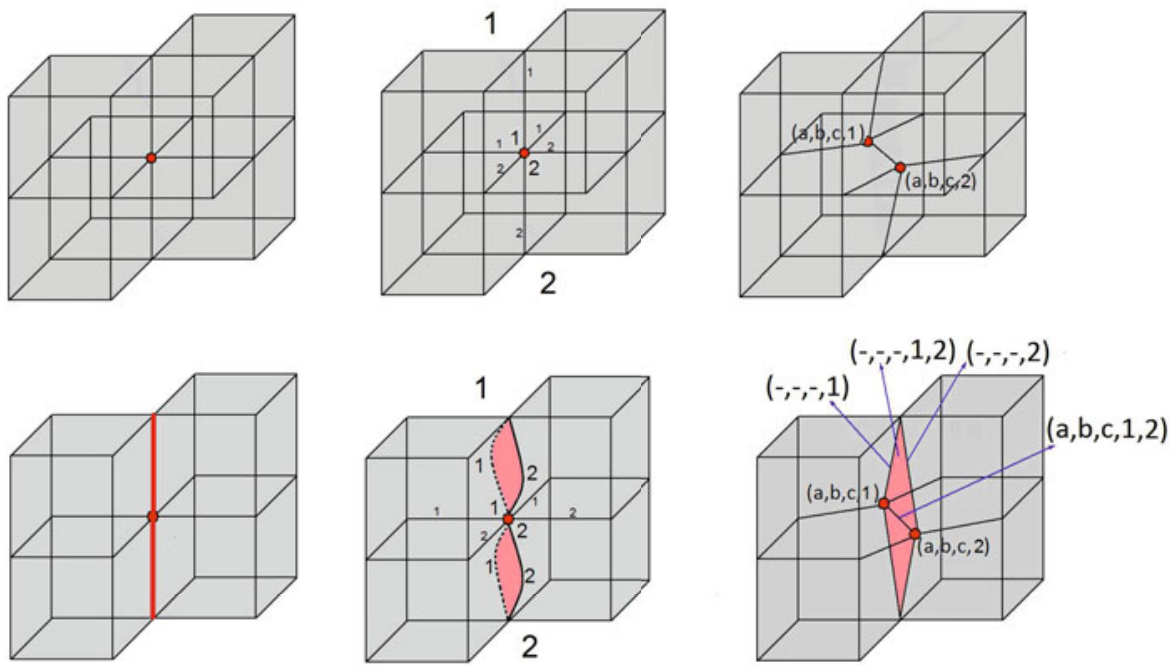

Fig. 6. Notations for the cells around a critical vertex $(a, b, c)$, with the configurations $C(6,1)$ (first row) and $C(4,1)$ (second one)
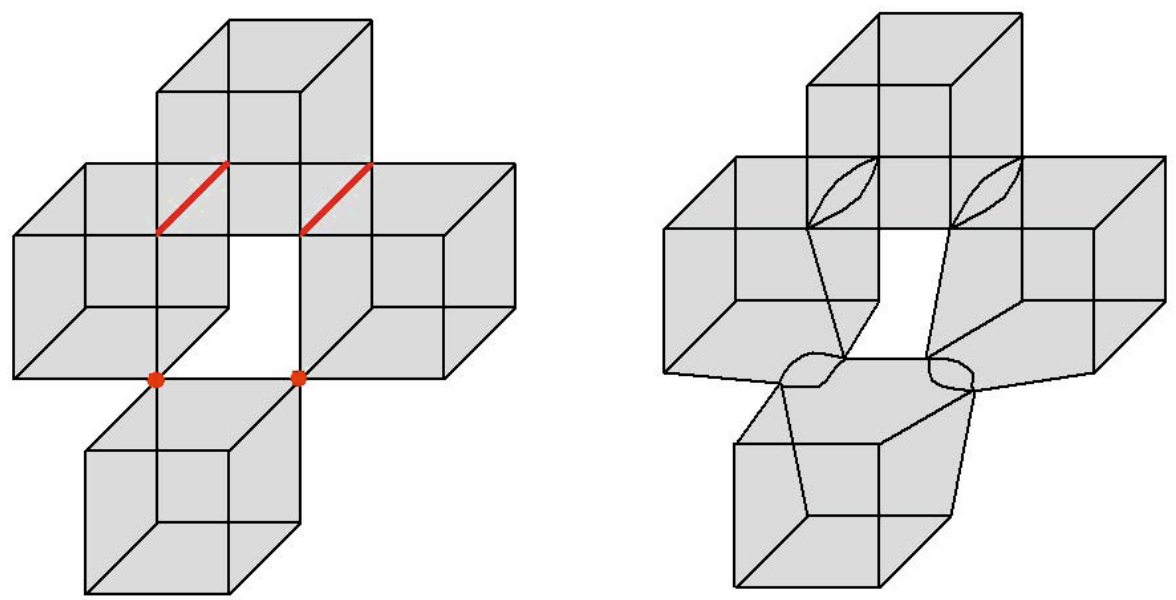

Fig. 7. Example of a cubical complex and well-composed complex

\section{Conclusion and Future Work}

We have presented a method for obtaining a well-composed cell complex from the cubical complex associated to a 3D binary digital image. We are convinced that this new representation will satisfy very nice properties: first, the new cell complex will allow to compute the homology of the image by computing the 
homology of the boundary surface of the cell complex; we will be able to geometrically control the representative cycles of homology generators in the sense that, for example, a 1-cycle will never belong to two different cavities (in fact, this could simplify the computation of the cup product in cohomology); moreover, algorithms developed for surfaces embedded in $\mathbb{R}^{3}$ ([1 $\left.\begin{array}{ll}1 & 2\end{array}\right]$ ) could be applied to the well-composed cell complex. Another future plan is to improve the notation used here to define the incidence index $k$ for the new cells.

\section{References}

1. Dey, T.K., Li, K.: Persistence-based handle and tunnel loops computation revisited for speed up. Computers \& Graphics 33, 351-358 (2009)

2. Dey, T.K., Li, K., Sun, J.: On computing handle and tunnel loops. In: IEEE Proceedings of the International Conference on Cyberworlds, pp. 357-366 (2007)

3. Dey, T.K., Li, K., Sun, J., Cohen-Steiner, D.: Computing Geometry-aware Handle and Tunnel Loops in 3D Models. ACM Transactions on Graphics 27(3), Article 45 (2008)

4. Gonzalez-Diaz, R., Jimenez, M.J., Medrano, B.: Cohomology ring of 3D cubical complexes. In: Proc. of the 13th International Workshop on Combinatorial Image Analysis, IWCIA 2009. Progress in Combinatorial Image Analysis, pp. 139-150 (2009)

5. Gonzalez-Diaz, R., Real, P.: Towards Digital Cohomology. In: Nyström, I., Sanniti di Baja, G., Svensson, S. (eds.) DGCI 2003. LNCS, vol. 2886, pp. 92-101. Springer, Heidelberg (2003)

6. Gonzalez-Diaz, R., Real, P.: On the Cohomology of $3 D$ Digital Images. Discrete Applied Math. 147, 245-263 (2005)

7. Gonzalez-Diaz, R., Jimenez, M.J., Medrano, B.: Cubical cohomology ring of 3D pictures. International Journal of Imaging Systems and Technologies (to appear)

8. Krahnstoever, N., Lorenz, C.: Computing curvature-adaptive surface triangulations of three-dimensional image data. Vis. Comput. 20(1), 17-36 (2004)

9. Kaczynski, T., Mischaikow, K., Mrozek, M.: Computational Homology. Applied Mathematical Sciences 157 (2004)

10. Latecki, L.J., Eckhardt, U., Rosenfeld, A.: Well-composed Sets. Comput. Vis. Image Underst. 61(1), 70-83 (1995)

11. Latecki, L.J.: 3D Well-Composed Pictures. Graphical Models and Image Processing 59(3), 164-172 (1997)

12. Latecki, L.: Discrete Representation of Spatial Objects in Computer Vision. Kluwer Academic, Dordrecht (1998)

13. Marchadier, J., Arques, D., Michelin, S.: Thinning grayscale well-composed images. Pattern Recognit. Lett. 25(5), 581-590 (2004)

14. Massey, W.S.: A basic course in algebraic topology. Springer, New York (1991)

15. Siqueira, M., Latecki, L.J., Tustison, N., Gallier, J., Gee, J.: Topological Repairing of 3D Digital Images. J. Math. Imaging Vis. 30, 249-274 (2008) 